\title{
ACTUALIZACIÓN DE TUMOR FIBROSO SOLITARIO PLEURAL. A PROPÓSITO DE UN CASO
}

Inmaculada Gómez-Molinero ${ }^{1 *}$; Olga Elena Tolentino-Ureña ${ }^{2}$; María Rosa Antón-Pastor y Beatriz de Pedro Huecas ${ }^{1}$ 'Centro de Salud Ciudad de los Periodistas, D.A. Norte, Hospital Universitario La Paz, Madrid, España; 2 Pontificia Universidad Católica Madre y Maestra, Facultad de Ciencias de la Salud, República Dominicana

RESUMEN: El tumor fibroso solitario (TFS) es una neoplasia de origen mesenquimal fibroblástico que puede aparecer en las distintas membranas serosas. Es una enfermedad rara que representa menos del $2 \%$ de todos los tumores de tejidos blandos. En este trabajo presentamos el caso de una paciente de 62 años con TFS pleural descubierto en el estudio de disnea como único síntoma, tratada con cirugía y dos años más tarde con quimioterapia por recidiva tumoral inoperable.

Palabras clave: Tumor pleural. Mesotelioma fibroso localizado.
UPDATE OF PLEURAL SOLITARY FIBROUS TUMOR. ABOUT A CASE

ABSTRACT: The solitary fibrous tumor (SFT) is a neoplasia of mesenchymal fibroblastic origin that can appear in different serous membranes. It is a rare disease that represents less than $2 \%$ of all soft tissue tumors. In this assignment, the case presented is a 62-year-old patient with pleural SFT which was discovered with only dyspnoea as a symptom; and was treated with surgery and 2 years later with chemotherapy because of inoperable tumor recurrence. Mex Med Fam. 2019;6:31-5)

Corresponding author: Inmaculada Gómez-Molinero, igomezmolinero@gmail.com

Key words: Pleural tumor. Localized fibrous mesothelioma.

\section{INTRODUCCIÓN}

El tumor fibroso solitario (TFS) es una neoplasia de origen mesenquimal fibroblástico que puede aparecer en las distintas membranas serosas: pleura, peritoneo y pericardio; también se ha descrito en mediastino, órbitas, senos paranasales y nasofaringe, tiroides, meninges y pulmón ${ }^{1}$.

Es una enfermedad rara. Representa menos del 2\% de todos los tumores de tejidos blandos. Los TFS de la pleura son los más frecuentes (2.8 por cada 100.000 individuos) y constituyen menos del 5\% de todos los tumores que surgen en la pleura ${ }^{2}$.

La presentación clínica puede iniciar con tos, disnea, dolor torácico (40-60\% de los pacientes) y de forma más excepcional como hemoptisis y neumonitis obstructiva por obstrucción de las vías respiratorias. En algunos casos se detecta incidentalmente una masa intratorácica en individuos asintomáticos ${ }^{3}$.

El diagnóstico es con pruebas de imagen y siempre precisa confirmación histológica. El
Correspondencia:

*Inmaculada Gómez-Molinero

E-mail: igomezmolinero@gmail.com
Fecha de recepción: 19-12-2018

Fecha de aceptación: 05-03-2019

DOI: 10.24875/RMF.19000129 


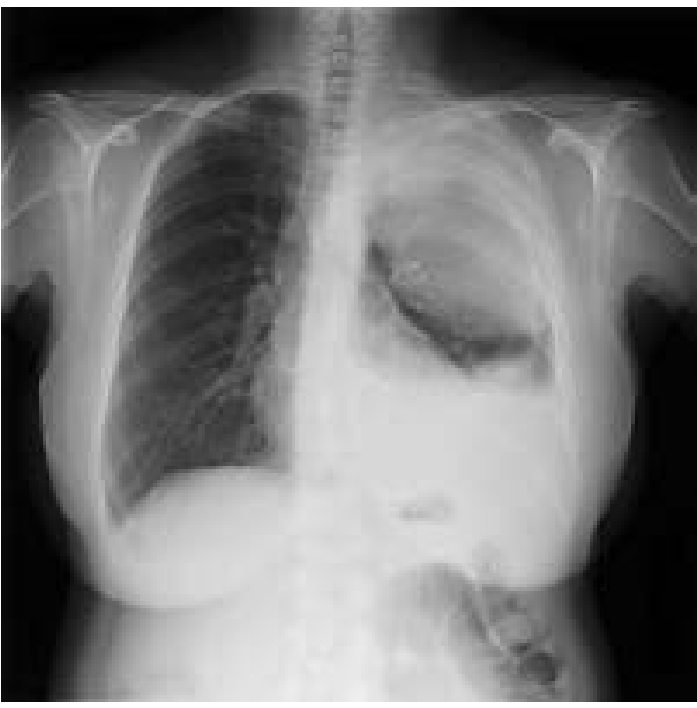

Figura 1. Radiografía posteroanterior de tórax.

diagnóstico diferencial radiológico del TFS pleural incluye hematomas extrapleurales, derrames pleurales loculados (pseudotumor pleural), metástasis pleurales, mesotelioma sarcomatoide y linfoma ${ }^{4}$.

Aunque la mayoría son tumores indolentes con alta tasa de supervivencia, todos los TFS pleurales y tienen potencial de recurrencia y de metástasis a sitios distantes ${ }^{5}$.

El manejo terapeútico de los TFS pleurales debe realizarse bajo la supervisión de comités de tumores multidisciplinarios. Incluye cirugía, quimioterapia y radioterapia. El seguimiento a largo plazo debe ser cuidadoso y deben participar profesionales de Atención Primaria y Especializada.

\section{PRESENTACIÓN DEL CASO}

Nuestra paciente es una mujer de 62 años que consulta a su médico de Atención Primaria por dificultad respiratoria tras esfuerzo físico de baja intensidad, de una semana de evolución, de instauración progresiva.

Entre sus antecedentes personales refiere ser fumadora de 20 paquetes/año, y bebedora ocasional de alcohol de 8 unidades semanales. En el resto de antecedentes personales y familiares no había datos de interés.

A la exploración física se objetiva una auscultación pulmonar anómala con hipofonía en hemitórax izquierdo como único

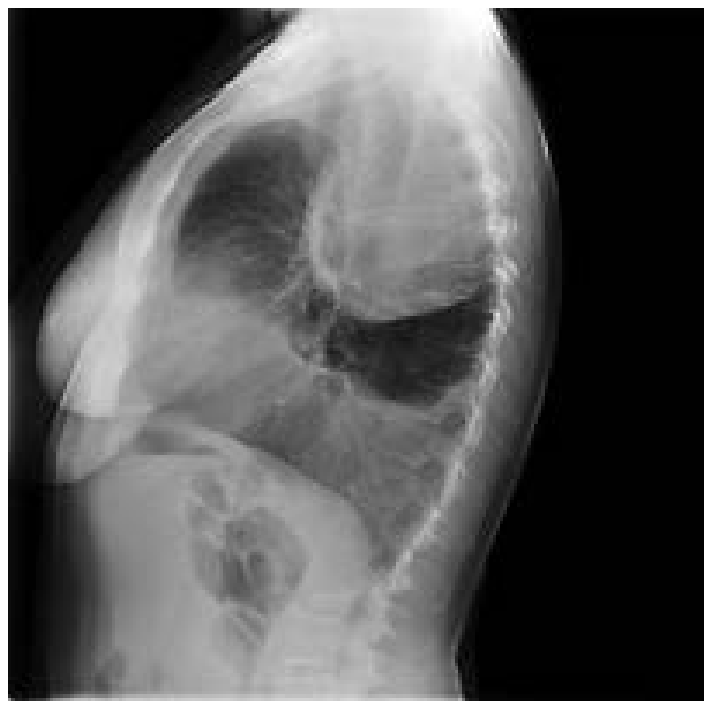

Figura 2. Radiografía lateral de tórax.

dato reseñable. Se solicita radiografía anteroposterior y lateral de tórax (Figs. 1 y 2) donde se objetiva masa de gran tamaño de probable origen extraparenquimatoso, en hemitórax izquierdo, que desplaza lóbulo superior y estructuras mediastínicas, asociado a derrame pleural moderado.

Se remite a la paciente al Servicio de Urgencias Hospitalarias, donde realizan toracocentesis evacuadora que mejora la disnea. Se remite la muestra obtenida a Laboratorio, Anatomía Patológica y Microbiología, con resultado de exudado negativo para células y marcadores tumorales, con un $10 \%$ de células mesenquimales. Estudio microbiológico e inmunológico negativo.

En el Departamento de Radiodiagnóstico realizan tomografía computarizada (TC) torácica que confirma la existencia de una masa de $14 \mathrm{~cm}$ de eje mayor, de aspecto heterogéneo que surge de la región pleural, comprimiendo el tejido pulmonar adyacente. Apuntan la posibilidad de TFS pleural a confirmar con estudio histológico.

La extirpación tumoral en el Servicio de Cirugía Torácica arroja una pieza tumoral pleural que se remite a Anatomía Patológica, donde describen una tumoración de $14 \mathrm{~cm}$ de bordes bien definidos con células de aspecto fusiforme con zonas pseudoquísticas y focos de necrosis que asienta en pleura 
parietal y visceral. Las células expresan en inmunohistoquímica CD34, Bcl-2 y CD99; y no expresan marcadores epiteliales (EMA), D2-40 ni calretinina. Se apunta como diagnóstico histológico: TFS pleural.

La paciente realiza seguimiento por Atención Primaria con su médico de familia y enfermera durante los meses posteriores a la cirugía, tratando las secuelas posquirúrgicas torácicas con analgésicos; educación sanitaria sobre el uso de inhaladores, fisioterapia respiratoria, vacunación antigripal y antineumocócica y monitorización de síntomas sospechosos de recidiva de forma trimestral.

El seguimiento en Neumología se realizó de forma semestral con valoración clínica y pruebas de imagen; la paciente permaneció sin recidivas durante dos años.

Aparece entonces nuevamente disnea de esfuerzo y se confirma recidiva de TFS en las pruebas diagnósticas realizadas TC torácica, biopsias pleurales por videotoracoscopia y tomografía por emisión de positrones - tomografia computarizada (PET-TC).

En el TAC de tórax se describe hemitórax izquierdo con múltiples implantes tumorales en pleura parietal, visceral y en mediastino. La prueba de tomografía por emisión de positrones y tomografia computarizada marcada con fluor 18 f-fluorodesoxiglucosa (18 F-FDG PET-TC) no evidenció lesiones hipercaptantes sospechosas de tejido tumoral maligno.

En Anatomía Patológica informan de que se trata de múltiples fragmentos de tejido pleural infiltrado por TFS con estudio de inmunohistoquímica STAT6 positivo.

Considerado el caso en el Comité de Tumores descartan la posibilidad de nuevo tratamiento quirúrgico y proponen tratamiento neoadyuvante con quimioterapia: cisplatino a dosis de $120 \mathrm{mg}$ i.v. y pemetrexed a dosis de $810 \mathrm{mg}$ i.v. en ciclos cada tres semanas, utilizando medicación complementaria para prevenir efectos secundarios con prehidratación (antes del cisplatino), dexametasona $16 \mathrm{mg}$ i.v. (continuando los tres días siguientes por v.o. con $8 \mathrm{mg}$ diarios), granisetrón $3 \mathrm{mg}$ i.v., ácido fólico $5 \mathrm{mg}$ diario v.o. y cianocobalamina $1,000 \mu \mathrm{g}$ i.m. de forma mensual.

\section{DISCUSIÓN}

El TFS fue descrito por primera vez en la pleura en 1931. Se le conoce con un número importante de nombres que hacen alusión a un supuesto origen mesotelial. El avance en técnicas diagnósticas de inmunohistoquímica (positivo para CD4 y vimentina, negativo para citoqueratinas, desmina y proteína S-100) permitió identificar el origen en células submesoteliales con características fibroblásticas y mesenquimatosas $^{6}$. En nuestra paciente el diagnóstico histológico se correspondía con estas características.

Los TFS son tumores raros, los más frecuentes asientan en pleura (2.8 por cada 100,000 individuos); son menos del 5\% de todos los tumores pleurales ${ }^{2}$.

La presentación clínica del TSF pleural se produce entre la $5 .^{a}$ y la $6 .{ }^{a}$ décadas de la vida, con clara preferencia por el sexo femenino (hasta el 70\%). Los datos de sexo y edad se corresponden con los de nuestra paciente (mujer de 62 años).

Los síntomas pulmonares inespecíficos (tos, disnea y dolor torácico) están presentes hasta en el 40-60\% de los pacientes. La presentación clínica en nuestro caso fue de disnea de esfuerzo.

Más raro es empezar con hemoptisis o neumopatía obstructiva. Hasta un $45 \%$ de los casos son asintomáticos y se detectan incidentalmente como una masa torácica en una radiografía de tórax solicitada por otros motivos ${ }^{7}$. Hasta el 20\% de los casos pueden estar asociados a dedos en palillo de tambor, osteoartropatía hipertrófica pulmonar por hipoxia crónica y secreción tumoral de ácido hialurónico, y otros síndromes paraneoplásicos tipo hipoglucemia refractaria por secreción tumoral de sustancias con acción insulinérgica, como factor de crecimiento II (Síndrome de Doege-Potter) ${ }^{8}$. El caso que presentamos corresponde a una mujer de 62 años con inicio clínico de disnea de esfuerzo. 
Dos tercios de los TFS pleurales asientan en la pleura visceral, unidos al pulmón por un pedículo. Un tercio se encuentran en la pleura parietal, siendo estos los de mayor tamaño (hasta $20 \mathrm{~cm}$ ), y con una amplia base de implantación. La paciente estudiada en nuestro caso presentaba un tumor gigante $(14 \mathrm{~cm})$ en pleura parietal $\mathrm{y}$, posteriormente, recidiva en ambas pleuras y en mediastino.

El diagnóstico de TFS puede ser sospechado por pruebas de imagen: radiografía de tórax, TC torácico y resonancia magnética nuclear de tórax, pero no existen características patognomónicas de este tipo de tumor. Suelen ser masas de tejido pleural bien circunscritas de aspecto homogéneo que pueden tener en su interior zonas quísticas, hemorragias y calcificaciones ${ }^{9,10}$. Tienden a desplazar, en lugar de invadir, a los tejidos circundantes. El derrame pleural es frecuente. Las imágenes radiológicas de nuestra paciente se muestran en las figuras 1 y 2 y cumplen los criterios descritos en la bibliografía.

La evaluación histopatológica del TFS pleural requiere una resección completa del tumor. El tamaño oscila entre 1 y $20 \mathrm{~cm}$, pero se han descrito piezas quirúrgicas de hasta $40 \mathrm{~cm}$. Son lesiones bien circunscritas con pseudocápsula o revestimiento seroso, a veces pedunculados y muy vascularizados. En su interior se pueden encontrar pseudoquistes, hemorragias y necrosis, particularmente en los más grandes. Los marcadores de inmunohistoquímica convencionales de TFS incluyen expresión de CD34, Bcl2, CD99 en ausencia de actina y EMA. La pieza quirúrgica de nuestro caso era de $14 \mathrm{~cm}$ con descripción anatomopatológica similar a la consultada en la revisión bibliográfica incluidos los datos de inmunohistoquímica ${ }^{11}$.

A nivel molecular los TFS se caracterizan por una inversión recurrente del brazo largo del cromosoma 12 (12q13) que da lugar a una fusión de dos genes, NAB2 y STAT6, que implican la diferenciación y proliferación celular, lo que favorece la tumorogénesis. En el TFS pleural se han descrito también mutaciones en el gen del factor de crecimiento de las plaquetas beta. No hay características moleculares que separen claramente los TFS benignos de los malignos ${ }^{12}$.

El pronóstico de los TFS pleurales depende de las recurrencias y de su potencial malignizante. En general son tumores indolentes con una supervivencia específica a los 10 años entre el 70 y el 100\%. La mayoría se comportan de manera benigna después de la resección completa, pero entre un 10 y un $25 \%$ recurren localmente y pueden metastatizar por diseminación local o hematógena a sitios distantes (pulmón, hígado, hueso y cerebro). La supervivencia prolongada es posible en los tumores resecables pero los pacientes con múltiples metástasis inoperables tienen peor pronóstico. Las recaídas tardías (10-40\%) se pueden producir hasta 20 años después de la presentación inicial; por eso es necesario un seguimiento continuo a largo plazo $^{13}$.

En el caso clínico descrito la recaída se produjo a los dos años de la resección tumoral completa, siendo la recurrencia local multifocal en pleura visceral, parietal y mediastino.

El tratamiento de todos los TFS, incluidos los pleurales, debe ser discutido por equipos multidisciplinarios con experiencia en esta enfermedad. Para los TFS localizados la resección quirúrgica completa es el pilar del tratamiento. En los TFS de múltiples localizaciones o inoperables no se recomienda radioterapia por la falta de eficacia descrita hasta el momento. Los tratamientos con quimioterapia no se recomiendan en tumores completamente resecados, pero hay que individualizar cada caso en el contexto de las recurrencias. En nuestra paciente un Comité de Tumores multidisciplinario tomó la decisión de no indicación quirúrgica y utilizar tratamiento neoadyuvante con quimioterapia.

Los agentes quimioterápicos utilizados en estos tumores son la ciclofosfamida, las antraciclinas $y$, más recientemente, inhibidores de la angiogénesis como el bevacizumab (con temozolomida) con respuesta 
terapéutica medida en «días libres de enfermedad» que no permite aconsejarla en todos los casos. Los pacientes deben ser alentados a participar en ensayos clínicos prospectivos, cuando estén disponibles, para evaluar la eficacia de nuevas terapias ${ }^{14}$. Nuestra paciente está siendo tratada en la actualidad de su recurrencia tumoral no susceptible de cirugía con ciclofosfamida y pemetrexel con seguimiento en el Servicio de Oncología Médica.

\section{CONCLUSIONES}

En Atención Primaria debemos conocer que el TFS pleural es una neoplasia poco común, usualmente asintomática, cuyo diagnóstico se presenta habitualmente como un hallazgo casual en una radiografía de tórax. El tratamiento de elección es la escisión quirúrgica completa $\mathrm{y}$, puesto que el comportamiento biológico es impredecible, el seguimiento de los casos debe enfocarse a la detección precoz de la recurrencia local o la aparición de metástasis.

El personal sanitario de Atención Primaria debe actualizar sus conocimientos en esta enfermedad para ayudar a sus pacientes en el seguimiento del proceso durante años asesorándoles en el manejo de la incertidumbre a largo plazo.

Con este caso podemos objetivar la importancia del equipo de Atención Primaria en el estudio de un paciente que consulta por disnea de esfuerzo realizando un diagnóstico diferencial mediante historia clínica, exploración física y pruebas complementarias adecuadas; coordinando la atención con los especialistas hospitalarios oportunos y acompañando al paciente durante todo el proceso, asesorándole en el manejo de la incertidumbre diagnóstica, orientándole sobre las posibilidades terapéuticas y aconsejándole un adecuado seguimiento de su enfermedad a lo largo de los años.

La actualización en enfermedades raras, como el TFS, compartida mediante publicaciones de acceso para los médicos de familia, nos permite realizar una buena educación para la salud desde Atención
Primaria, donde tanto el paciente como sus familiares aprenden los diferentes abordajes en este tipo de tumores, así como sus complicaciones más frecuentes y su pronóstico a largo plazo. Les enseñamos a descubrir precozmente las recidivas monitorizando los síntomas de alarma. De esta manera empoderamos al paciente en el seguimiento de su enfermedad y logramos más apego al cumplimiento de sus visitas programadas en Atención Primaria.

\section{DECLARACIÓN DE CONFLICTO DE INTERESES}

Los autores declaran no presentar ningún conflicto de intereses para la publicación de este manuscrito.

\section{BIBLIOGRAFÍA}

1. Bar I, Papiashvilli M, Zukerman B, Stav D, Sandbank J. Large solitary fibrous tumour of the pleura: analysis of six cases. Heart Lung Circ. 2007;16(4):282-4.

2. Briselli M, Mark EJ, Dickersin GR. Tumores fibrosos solitarios de la pleura: ocho nuevos casos y revisión de 360 casos en la literaturáncer. 1981:47:2678.

3. Tapias LF, Mercier O, Ghigna MR, et al. Validación de un sistema de puntuación para predecir la recurrencia de tumores fibrosos solitarios resecados de la pleura. Chest. 2015;147:216.

4. Walker CM, Takasugi JE, Chung JH, et al. Condiciones tumorales de la pleura. Radiografías. 2012;32:971.

5. Cranshaw IM, Gikas PD, Fisher $C$, et al. Resultados clínicos de los tumores fibrosos solitarios extra torácicos. Eur J Surg Oncol. 2009;35:994.

6. Granville L, Laga AC, Allen $T C$, et al. Review and update of uncommon primary pleural tumors: a practical approach to diagnosis. Arch Pathol Lab Med. 2005;129(11):1428-43.

7. Sánchez-Mora N, Cebollero-Presmanes M, Monroy V, CarreteroAlbiñana L, Herranz-Aladro M, Álvarez-Fernández E. Tumor fibroso solitario pleural: características clinicopatológicas de una serie de casos y revisión de la bibliografía. Arch Bronconeumol. 2006;42(2):96-9.

8. Tominaga N, Kawarasaki C, Kanemoto K, et al. Tumor fibroso solitario recidivante de la pleura con transformación maligna y hipoglucemia inducida por tumor no celular de islote debido a sobreexpresión paraneoplásica y secreción de factor II de crecimiento similar a insulina de alto peso molecular. Intern Med. 2012;51:3267.

9. Saifuddin A, Da Costa P, Chalmers AG, et al. Tumores fibrosos localizados primarios malignos de la pleura: características clínicas, radiológicas y patológicas. Clin Radiol. 1992;45:13.

10. Inglaterra DM, Hochholzer L, McCarthy MJ. Tumores fibrosos benignos y malignos localizados de la pleura. Una revisión clinicopatológica de 223 casos. Am J Surg Pathol. 1989;13:640.

11. Schirosi L, Lantuejoul S, Cavazza A, et al. Tumores fibrosos pleuro-pulmonares solitarios: estudio clinicopatológico, inmunohistoquímico y molecular de 88 casos confirmando el valor pronós tico del sistema de estadificación de Perrot y la expresión de p53 y evaluando el papel de c-kit, BRAF, PDGFRs (alfa/beta), c-met y EGFR. Am J Surg Pathol. 2008;32:1627.

12. Mohajeri A, Tayebwa J, Collin A, et al. El análisis genético integral identifica un gen de fusión patognomónico NAB2/STAT6, desequilibrios genómicos secundarios no aleatorios y un perfil de expresión génica característico en el tumor fibroso solitario. Genes Cromosomas Cáncer. 2013;52:873.

13. Park CK, Lee DH, Park JY, et al. Tumores fibrosos solitarios malignos recurrentes múltiples: seguimiento a largo plazo de 24 años. Ann Thorac Surg. 2011;91:1285.

14. Stacchiotti $S$, Tortoreto $M$, Baldi GG, et al. Evidencia preclínica y clínica de la actividad de pazopanib en el tumor fibroso solitario. Eur J Cancer. 2014;50:3021. 